\title{
Space Variant PSF - Deconvolution of Wide-Field Astronomical Images
}

M. Řeřábek

\begin{abstract}
The properties of UWFC (Ultra Wide-Field Camera) astronomical systems along with specific visual data in astronomical images contribute to a comprehensive evaluation of the acquired image data. These systems contain many different kinds of optical aberrations which have a negatively effect on image quality and imaging system transfer characteristics, and reduce the precision of astronomical measurement. It is very important to figure two main questions out. At first: In which astrometric depend on optical aberrations? And at second: How optical aberrations affect the transfer characteristics of the whole optical system. If we define the PSF (Point Spread Function) [2] of an optical system, we can use some suitable methods for restoring the original image. Optical aberration models for LSI/LSV (Linear Space Invariant/Variant) [2] systems are presented in this paper. These models are based on Seidel and Zernike approximating polynomials [1]. Optical aberration models serve as suitable tool for estimating and fitting the wavefront aberration of a real optical system. Real data from the BOOTES (Burst Observer and Optical Transient Exploring System) experiment is used for our simulations. Problems related to UWFC imaging systems, especially a restoration method in the presence of space variant PSF are described in this paper. A model of the space variant imaging system and partially of the space variant optical system has been implemented in MATLAB. The "brute force" method has been used for restoration of the testing images. The results of different deconvolution algorithms are demonstrated in this paper. This approach could help to improve the precision of astronomic measurements.
\end{abstract}

Keywords: High order aberrations, PSF, UWFC, BOOTES, Zernike, Seidel, LSI, LSV, deconvolution, image processing.

\section{Introduction}

A wide range of applications use lenses with a wide angle of view (WFC, UWFC) for sky monitoring. Detection of new objects e.g. novae, supernovae and AGN (Active Galactic Nuclei) is a well known application in which these images are analysed. All-sky imaging (monitoring) based on so-called "fish-eye" lenses is also used in some applications.

This paper deals with scientific (astronomical) image data processing. Real image data from the BOOTES experiment and from double-station video observation of meteors is analyzed. BOOTES is a system for monitoring the optical transient of GRB (Gamma Ray Bursts). The main goal of double-station video observation of meteors to acquire and analyse video records of meteors. The images from these systems contain survey data with huge numbers of objects of small size. BOOTES is an automatic robotic system located in southern Spain. It is equipped with a set of telescopes and CCD (Charged-Couple Device) cameras, which are fitted with lenses of various focal lengths. Each camera is used for a different task in monitoring the OT (optical transient) of the GRB and AGN. In the following text, we deal with image data acquired from the UWFC optical system. The second application is double-station video observation of meteors in the Czech Republic. Observation using a double camera system enables us to determine the trajectory of meteors in the earth's atmosphere. UWFC is also used in this system. High quality of the imaging system is therefore required.

UWFC image data analysis is very difficult in general. There are many different kinds of optical aberrations and distortions in these systems. Moreover, the objects in ultra wide-field images are very small (a few pixels per object dimension). Optical aberrations have their greatest impact toward the margins of the FOV (Field of View), it means that aberration error growing with increasing angular distance from optical axis of the system. These aberrations distort the PSF of the optical system and rapidly reduce the accu- racy of the astrometry measurements. Optical aberrations are dependent on three-dimensional coordinates. This relation affects the transfer characteristics of optical systems and makes them spatially variant. The influence of spatially variant optical aberrations on the transfer function of optical imaging systems is outlined in this paper.

\section{Transfer characteristics of optical imaging systems}

The description of transfer characteristics (mainly PSF) of optical imaging system is presented in the following section. To specify the properties of the lens optical system, we adopt the point of view that all optical elements are lumped into a "black box". Referring to Fig. 1, the terminals of this black box consist of the planes containing the entrance and exit pupils [2].

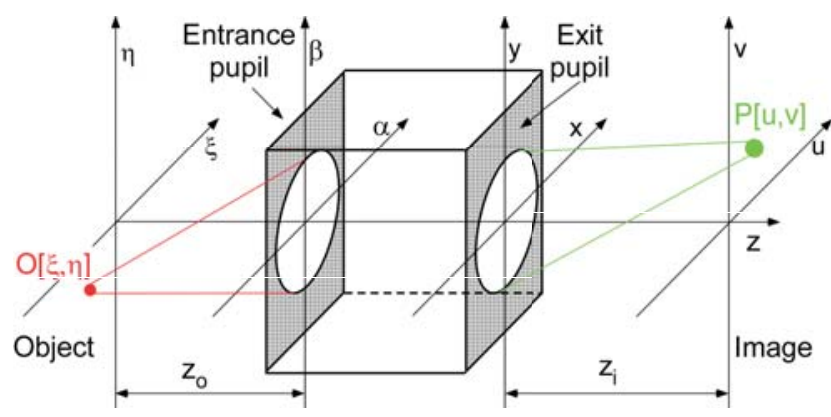

Fig. 1: Generalized model of an optical imaging system

\subsection{Point Spread Function}

The smallest detail that the imaging system can produce is determined by its impulse response $h(\xi, \eta)$. The impulse response, referred to in optical systems as the point spread 
function (PSF), describes spatial distribution of the illumination in the image plane, when the point source in object plane is used. PSF is actually the response of the optical imaging system to the two dimensional Dirac impulse (see Fig. 2).

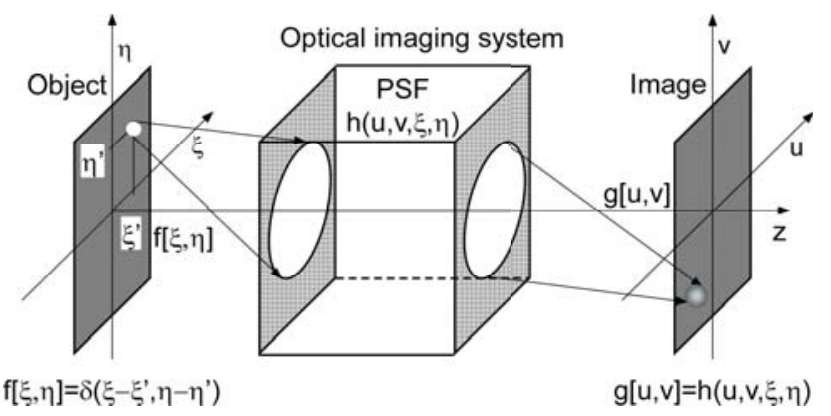

Fig. 2: Two dimensional impulse response of the optical imaging system - i.e. point spread function (PSF)

\section{Effects of aberrations on PSF}

Optical systems with all aberrations compensated are known as diffraction limited system. The influence of aberrations on image quality can be expressed as the wavefront error at the exit pupil, and their effects on the transfer characteristics can be expressed as the changes in the PSF size and shape. When an imaging system is diffraction limited, the PSF consists of the Fraunhofer diffraction pattern of the exit pupil [2]. In that case, we consider the departures of the exit-pupil wavefront from ideal spherical form.

\subsection{Shift invariant PSF modeling}

The relation between the object and image of an LSI optical system can be expressed by the convolution in the spatial domain (the object irradiance distribution $f(\xi, \eta)$ is convolved with the impulse response $h(u, v))$ [5]. The PSF of the LSI optical imaging system can be expressed as

$$
\begin{aligned}
& \operatorname{PSF}(x, y)=|F T\{q(u, v)\}|^{2} \\
& =\left|F T\left\{p(x, y) \exp \left(-i \frac{2 \pi}{\lambda} W(x, y)\right)\right\}\right|^{2},
\end{aligned}
$$

where $p(x, y)$ defines the shape, size and transmission of the exit pupil, and $\exp [-i 2 \pi / \lambda(W(x, y))]$ accounts for the phase deviation of the wavefront from a reference sphere. Fig. 3 shows the PSF of diffraction limited optical system. An example of coma aberration (one of the basic optical aberration) $[1,3]$ influence to PSF is presented in Fig. 4.

\subsection{Shift variant PSF modeling}

When the impulse response of the optical imaging system depends on the coordinates of the object $(\xi, \eta)$, we speak about Linear Shift Variant (LSV) systems. If we consider the LSV system, we cannot use convolution to express the relation between object and image. In order to compute PSF we have to use the diffraction integral $[1,3,4]$. PSF can then be expressed as $[6,10]$

$$
\begin{aligned}
& h(u, v, \xi, \eta)= \\
& \frac{1}{\lambda^{2} z_{0} z_{i}} \int_{-\infty-\infty}^{\infty} \int_{-\infty}^{\infty} p(x, y) \exp \left(-i \frac{2 \pi}{\lambda} W(x, y)\right) \ldots \\
& \ldots \exp \left\{-j \frac{2 \pi}{\lambda z_{i}}[(u-M \xi) x+(u-M \eta) y]\right\} \mathrm{d} x \mathrm{~d} y .
\end{aligned}
$$

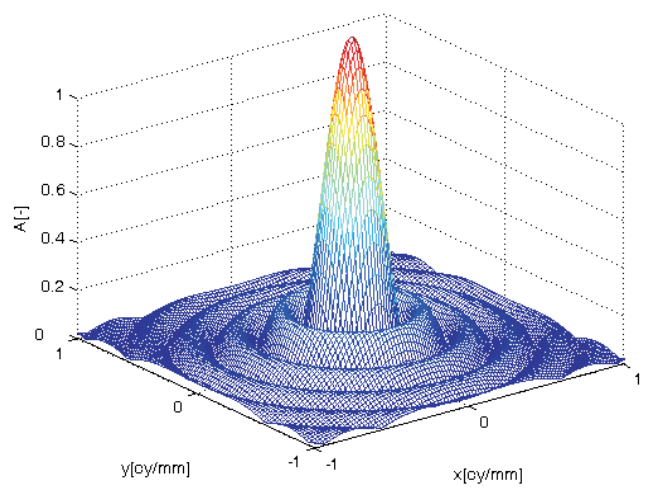

Fig. 3: PSF of a diffraction limited (aberration free) system

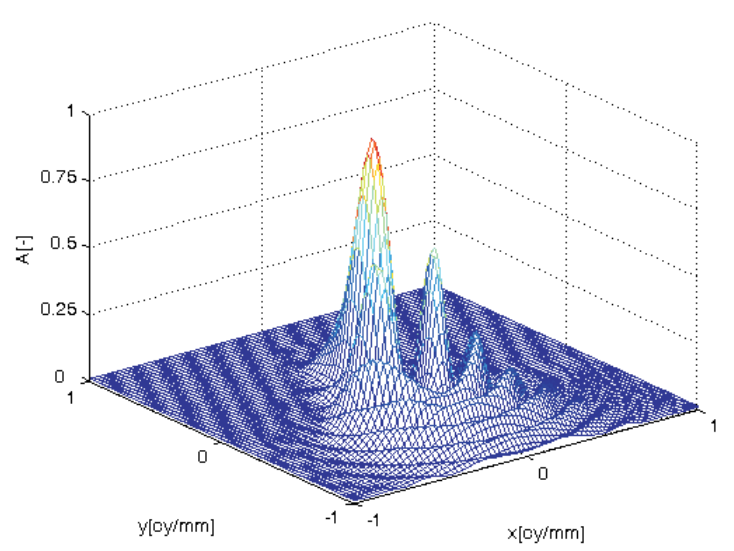

Fig. 4: PSF distorted with coma aberration

Fig. 5 shows real astronomical data, which was taken within the double-station observation project. This program is currently running at the Ondřejov Observatory. A stellar object profile $[8,9]$ depending on the image position is also demonstrated in Fig. 5.

\section{Model of the spatially variant imaging system}

Astrometric measurements are often limited by variations in PSF shape and size over the image. These variations in PSF structure occur especially in UWFC systems, because of the amount of aberrations. Optical aberrations increase toward the margins, as mentioned above. The principal difficulty in spatially variant (SV) systems is that Fourier approaches can no longer be used for restorations (deconvolutions) of the original image [5, 7].

Let us consider the SV optical system distorting only by coma aberration. If we want to use Fourier methods for the 

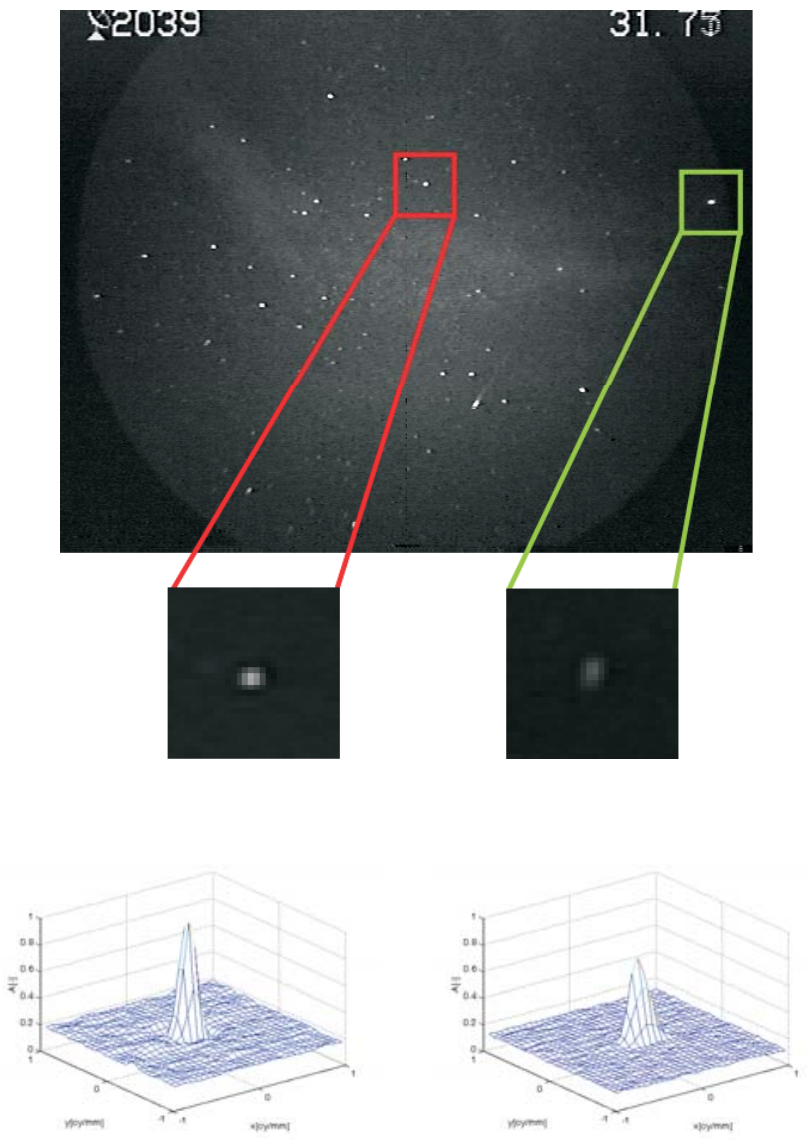

Fig. 5: Input image data from the system at the Ondřejov base. Demonstration of the influence of aberrations at the edge and in the centre of an image.

deconvolution process, we need to split the original image. The transfer characteristics of each part (so called isoplanatic patch) are described by a unique PSF. This system is called a partially space invariant system, and we use it in our experiments (see Fig. 6).

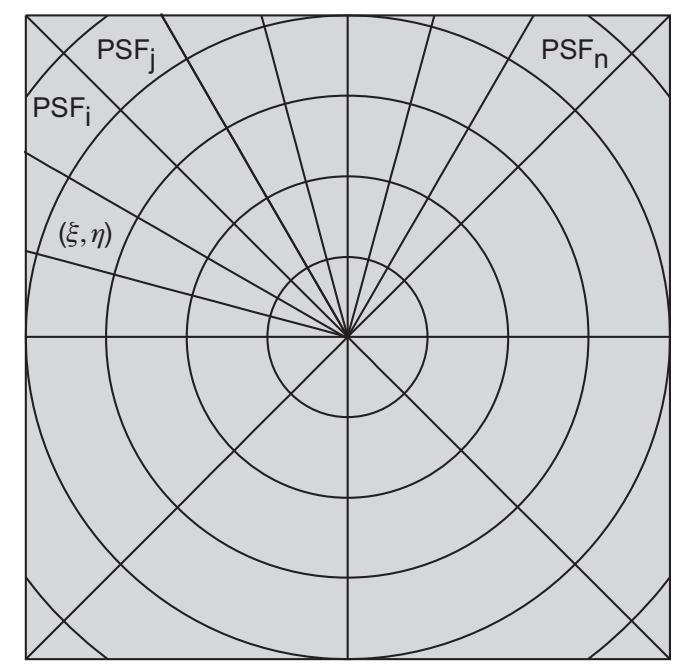

Fig. 6: Model of a partially space invariant optical system

Such a system has parametric PSF - for each value of a parameter (in our case it is the coordinate of the object in the object plane) the PSF takes a different size and shape according to the aberrations.

The wavefront aberration function for the SV optical system can be described as

$$
W(\rho, \theta, \delta, \varphi)=\sum_{n}^{k} \sum_{m=-n}^{n} W_{n(\delta, \varphi)}^{m} Z_{n}^{m}(\rho, \varphi-\theta),
$$

where $W_{n(\delta, \varphi)}^{m}$ is the RMS wavefront error for aberration mode ${ }_{n}^{m}$ and for object coordinate $(\delta, \varphi)$.

Use of the partially space invariant system enables us to describe the transfer characteristics by Fourier methods. The PSF of this system can then be described as

$\operatorname{PSF}(u, v, \delta, \varphi)=\left|F T\left\{p(x, y) \exp \left(-i \frac{2 \pi}{\lambda} W(\rho, \theta, \delta, \varphi)\right)\right\}\right|^{2}$

where $(\delta, \varphi)$ is the polar coordinate on the object plane. So we obtain a number of PSFs, one for each part. Now, we can also use the Fourier approach for image deconvolution.

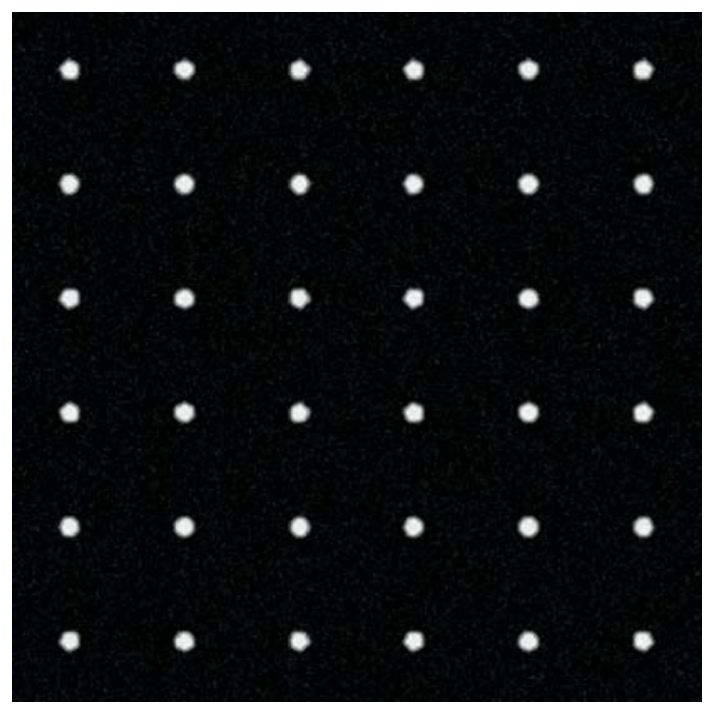

a)

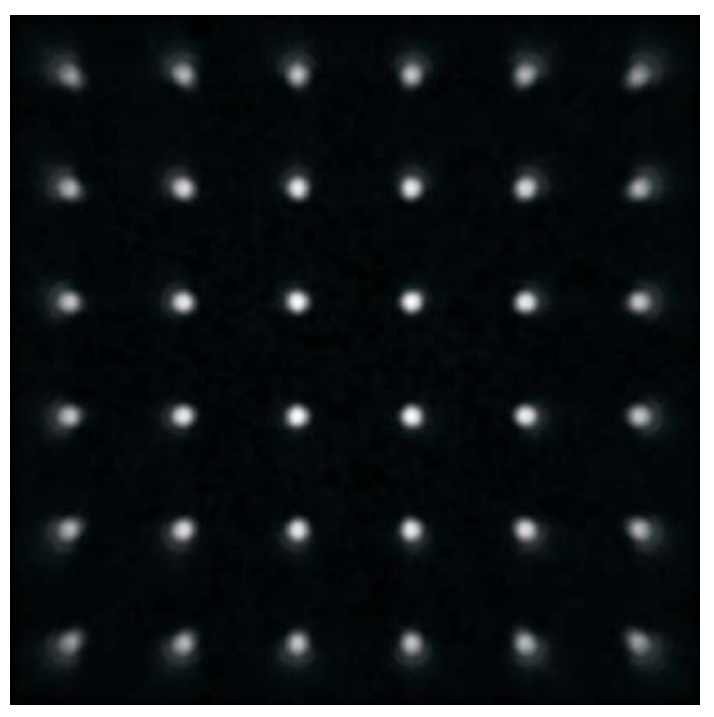

b)

Fig. 7: a) Source image - object, b) Image passed through the SV coma distorted optical system 


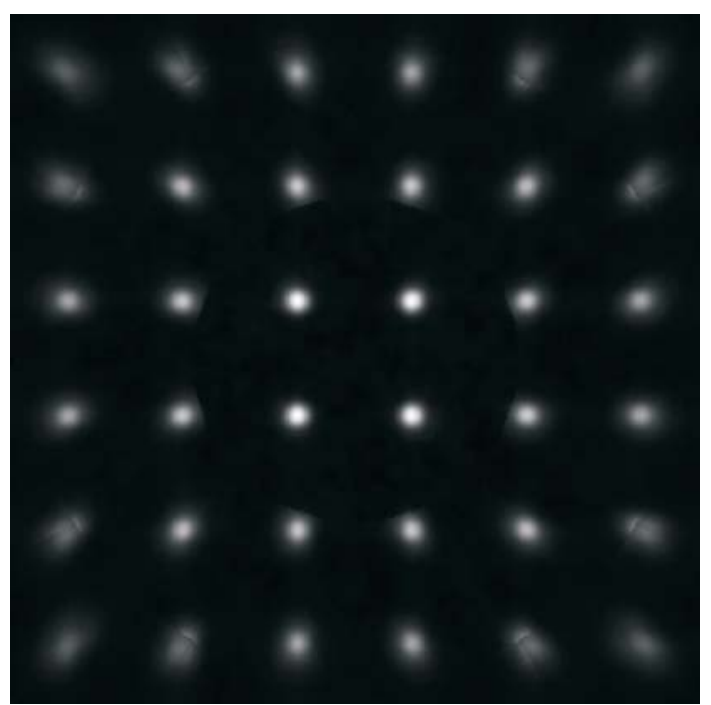

a)

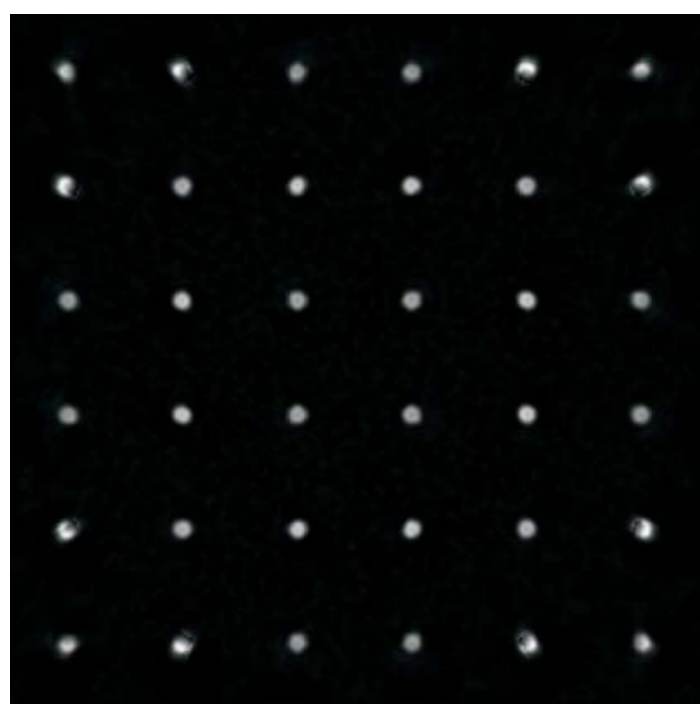

b)

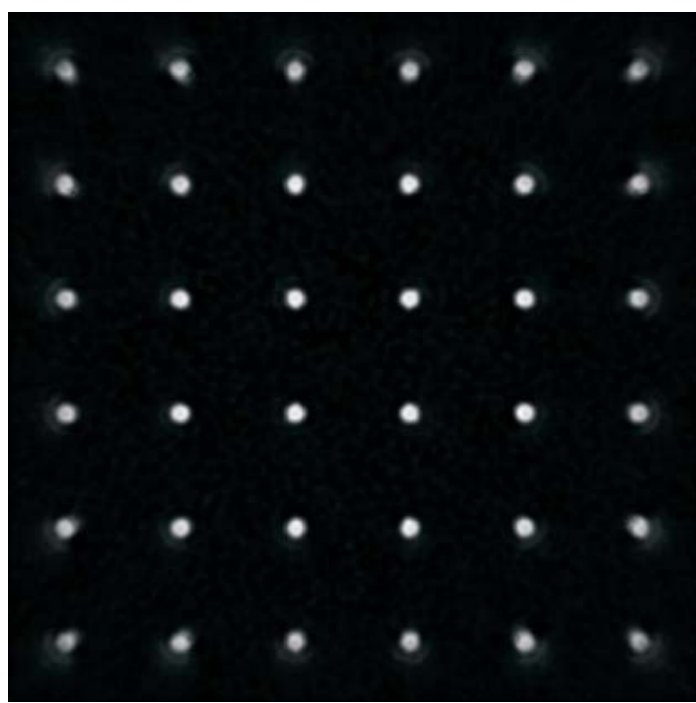

C)

Fig. 8: Restored images: a) Wiener deconvolution. b) Lucy - Richardson deconvolution. c) Blind deconvolution
The optical system is divided into 40 spatially invariant parts in our experiments. Three deconvolution algorithms [8, 9] - Wiener, Lucy-Richardson and Blind -have been used for restoring the original image (see Fig. 7 and 8).

The Wiener deconvolution algorithm gives inaccurate results. This method would need finer divisions. The Lucy-Richardson method gives the best results. The results are influenced by the setting of the weighting function of each spatially invariant part.

\section{Conclusions}

Optical aberration models for LSI, LSV and partially invariant systems have been presented in this paper. These models are based on the Seidel and Zernike approximating polynomials. We can commonly use these approaches for modeling the WFC and UWFC of the BOOTES experiment and also for double-station video observation of meteors. The influences of coma aberration on astrometric measurement precision have been shown. The effect of optical aberrations and spatial variations on the transfer function (PSF) of optical imaging systems have been described. The results of various deconvolution algorithms have been demonstrated.

The goal of our future work is to find the PSF model of high order optical aberrations for real SV (partially space invariant system) and to remove aberrations from the image. It will be necessary to find sufficient splitting of the spatial variant system, and to find a proper deconvolution method for removing the aberrations. This approach will also help to improve the precision of astronomic measurements around the optical axis and on the edges of FOV (far from the optical axis).

\section{Acknowledgments}

The research described in this paper was supervised by Mgr. Petr Páta, PhD., FEE CTU in Prague. This work has been supported by grant No. CTU0707613 "Shift Variant Optical Systems in Imaging Algorithms" of CTU in Prague.

A part of this research work has been supported by the Czech Grant Agency under grant No. 102/05/2054 “Qualitative Aspects of Audiovisual Information Processing in Multimedia Systems".

\section{References}

[1] Born, M., Wolf, E.: Principles of Optics. $2^{\text {nd }}$ ed. London: Pergamon Press, 1993.

[2] Goodman, J. W.: Introduction to Fourier Optics. Boston: McGraw-Hill, 1996.

[3] Hopkins, H. H.: Wave Theory of Aberrations. London, Oxford University Press, 1950.

[4] Goodman, J. W.: Linear Space-Variant Optical Data Processing. Optical Information Processing, edited by S. H. Lee, Berlin, Heidelberg, New York: Springer-Verlag, 1981, p. 235-260. ISBN 3-540-10522-0.

[5] Lohman, A. W., Paris, D. P.: Space-Variant Image Formation. Journal of the Optical Society of America, Vol. 55 (1965), No. 8, p. 1007-1013.

[6] Campisi, P., Egiazarian, K.: Blind Image Deconvolution: Theory and Applications. CRC, 2007. 
[7] Řeřábek, M., Páta, P., Koten, P.: High Order Optical Aberration Influence to Precision of Astronomical Image Data Processing. In SPIE Europe Conference Proceedings 6584. Prague, 2007. ISBN: 9780819467126

[8] Starck, J., Murtagh, F.: Astronomical Image and Data Analysis. Berlin: Springer Verlag, 2002.

[9] Starck, J., Murtagh, F., Bijaoui, A.: Image and Data Analysis: The Multiscale Approach. Cambridge University Press, 1998.

[10] Maeda, P. Y.: Zernike Polynomials and Their Use in Describing the Wavefront Aberrations of the Human Eye. 2003.

\section{Martin Řeřábek}

e-mail: rerabem@.fel.cvut.cz

Department of Radioelectronics

Czech Technical University in Prague

Faculty of Electrical Engineering

Technická 2

16627 Praha, Czech Republic 